\title{
Optical power-based interrogation of plasmonic tilted fiber Bragg grating biosensors
}

\author{
Á. González-Vila*a , A. Lopez-Aldaba ${ }^{\mathrm{b}}$, D. Kinet ${ }^{\mathrm{a}}$, P. Mégret ${ }^{\mathrm{a}}$, M. Lopez-Amo ${ }^{\mathrm{b}}$, C. Caucheteur ${ }^{\mathrm{a}}$ \\ ${ }^{\mathrm{a}}$ Electromagnetism and Telecommunication Dept., University of Mons, \\ Boulevard Dolez 31, B-7000 Mons, Belgium \\ ${ }^{\mathrm{b}}$ Dept. of Electrical and Electronic Engineering, Universidad Pública de Navarra, \\ Campus Arrosadia S/N, E-31006 Pamplona, Spain
}

\begin{abstract}
Two interrogation techniques for plasmonic tilted fiber Bragg grating sensors are reported and experimentally tested. Typical interrogation methods are usually based on tracking the wavelength shift of the most sensitive cladding mode, but for biosensing applications, spectrometer-based methods can be replaced by more efficient solutions. The proposed techniques thus rely on the measurement of the induced changes in optical power. The first one consists of a properly polarized tunable laser source set to emit at the wavelength of the sensor most sensitive mode and an optical power meter to measure the transmitted response. For the second method, a uniform fiber Bragg grating is photo-inscribed beyond the sensor in such a way that its central wavelength matches the sensor most sensitive mode, acting as an optical filter. Using a LED source, light reflected backwards by this grating is partially attenuated when passing through the sensor due to plasmon wave excitation and the power changes are quantified once again with an optical power meter. A performance analysis of the techniques is carried out and they both result competitive interrogation solutions. The work thus focuses on the development of cost-effective alternatives for monitoring this kind of biosensors in practical situations.
\end{abstract}

Keywords: Optical fiber sensors, fiber Bragg grating, tilted fiber Bragg grating, sensor interrogation, surface plasmons

\section{INTRODUCTION}

Surface plasmon resonance (SPR) sensors comprise an excellent tool to measure refractive index changes in dielectric media and are especially suitable for the detection of chemical interactions. Surface plasmon waves consist in collective oscillations of electrons at a metal-dielectric interface and the most typical platform in which they have been explored is the well-known Kretschmann prism ${ }^{1}$. With this configuration, a light beam is transmitted through a high index prism and reaches one of its external faces, which has a metallic film attached. For a certain incident angle, a surface plasmon wave is excited at the interface between the metallic layer and the medium on top. This way, changes in the refractive index of the medium cause a shift of the angle with which the light is reflected and then transmitted out of the prism.

Among the optical fiber-based solutions developed to outperform bulky SPR platforms, plasmonic tilted fiber Bragg grating (TFBG) sensors ${ }^{2}$ do not require to modify the optical fiber geometry and they allow to use telecommunicationgrade equipment. A TFBG is a periodic modulation of the refractive index of the core of an optical fiber, which is angled with respect to the perpendicular to the fiber longitudinal axis. It couples light propagating through the core into multiple cladding modes, each of them with a corresponding effective refractive index value. Thus, as soon as this value approaches the one of the surrounding medium, the cladding modes get coupled out of the fiber, producing a change in the grating transmitted spectrum. Although TFBGs are inherently highly accurate refractometers ${ }^{3}$, their sensitivity can be enhanced through a thin metallic coating. This permits to excite a surface plasmon wave at the interface between the metallic film and the outer medium when the input light is linearly polarized in the plane of the grating tilt direction.

In biosensing applications plasmonic TFBG sensors usually work in similar media ${ }^{4}$, e.g. phosphate-buffered saline (PBS) or blood, which involves the same refractive index region and thus their analysis is carried out on the same sensitive mode. Hence, for cost-effective reasons, there is no need for a typical spectrometer-based interrogation and the change in optical power ${ }^{5}$ can also be measured using a narrow-band demodulation technique. In this work, two power-based interrogation techniques are proposed, relying on both the transmitted and reflected optical power. The aim is to provide competitive solutions for the monitoring of these optical platforms in real-life scenarios.

*alvaro.gonzalezvila@umons.ac.be; phone+32 (0) 653743 67; fax +32 (0) 653741 99; umons.ac.be/telecom 


\section{EXPERIMENTAL SETUPS}

For the fabrication of the sensor used in the following experiments a $6^{\circ}$ TFBG was first photo-inscribed in the core of a hydrogen-loaded photosensitive optical fiber (FiberCore PS-1250). The inscription was carried out by UV irradiation from a continuous wave (CW) frequency-doubled Ar-laser emitting at $244 \mathrm{~nm}$ and finally passing through a phase mask with a pitch of $1070 \mathrm{~nm}$, before reaching the fiber. Both the mask and the fiber were placed perpendicularly to the writing beam, but the first was rotated around the axis of the beam, in order to get the desired tilted pattern on the grating planes. The TFBG was next kept into an oven at a temperature of $85^{\circ} \mathrm{C}$ for about 12 hours to stabilize its behavior at room temperature. Finally, a gold thin film with a thickness of $50 \mathrm{~nm}$ was deposited around the grating ${ }^{6}$ by means of a sputtering process (Leica EM SCD 500). After sensor fabrication, it was immersed into a solution consisting of distilled water and lithium chloride $(\mathrm{LiCl})$ so the plasmon wave could be excited in an aqueous environment.

\subsection{Narrow-band interrogation based on the transmitted optical power}

The gold coating deposited around the optical fiber at the TFBG location defines a metal-dielectric interface between the metallic film and the solution in which the sensor was immersed. When properly polarized cladding modes reach this interface, a surface plasmon wave is excited, producing a characteristic shape on the transmitted spectrum. As it can be seen in Figure 1a, the SPR mode is highlighted, while the adjacent modes on its left are the ones sensitive to refractive index changes in the surrounding medium. In this case, the most sensitive mode is the one illustrated in Figure 1b, which exhibits the most significant change in optical power.

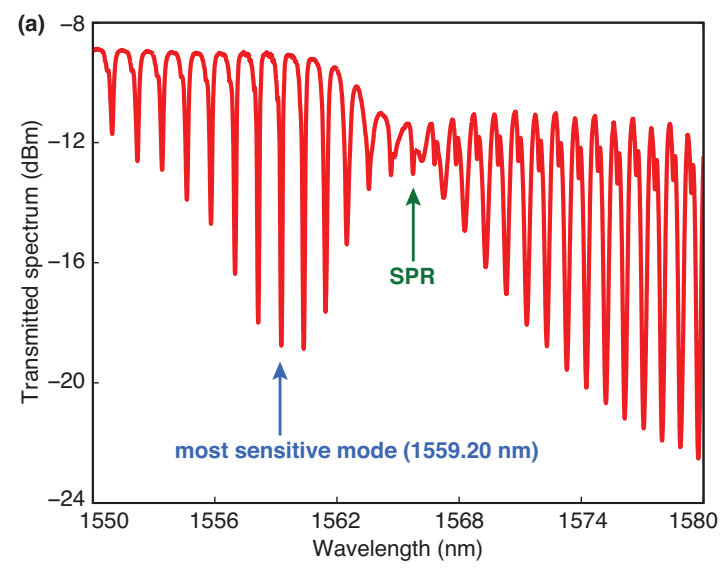

(b)

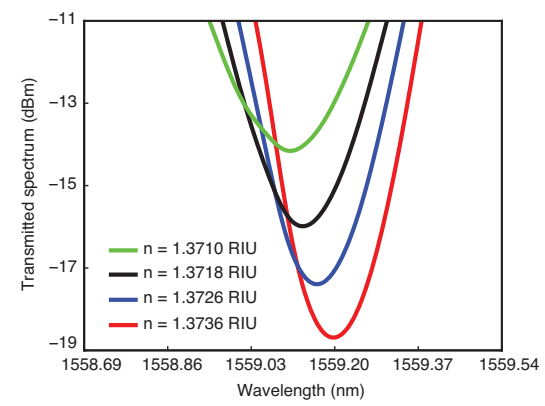

(c)

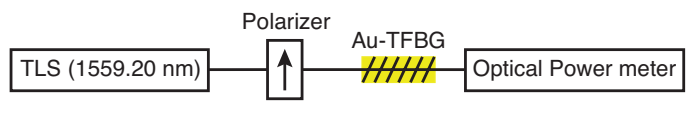

Figure 1. (a) Transmitted spectrum of the plasmonic TFBG sensor, (b) behavior of the most sensitive mode with respect to surrounding refractive index changes and (c) experimental setup for the transmitted power-based technique.

The basis of the first interrogation technique is to profit from this power change produced on the transmitted spectrum to measure the changes in the refractive index of the outer solution. As it is schematized in Figure 1c, the setup consists of a tunable laser source (TLS) configured to emit at the wavelength of the most sensitive mode, that is radially polarized before reaching the sensor, whose response is finally measured with an optical power meter (dBm Optics Model 4100). In this way, the measured transmitted power is proportional to the refractive index of the medium.

\subsection{Interrogation based on the optical power reflected by a FBG located just beyond the sensor}

For the second technique, the same mode is interrogated in terms of optical power change but some modifications are carried out to be able to perform the measurements in reflection. As shown in Figure 2, a uniform FBG is first photoinscribed just after the sensor through Lloyd's mirror interferometry ${ }^{7}$ to finely establish its central wavelength and match it to the one of the aforementioned mode, acting as an optical filter. What is obtained is thus a wavelength-gated ${ }^{8}$ response composed by the reflection spectrum of the FBG that is partially attenuated by the most sensitive mode of the sensor. In this way, the surrounding refractive index changes will produce a variation of the optical power comprised inside the main lobe of the FBG. As in this case a broadband analysis is performed, a LED source (Amonics ALS-CL17-B-FA) was used, together with a linear polarizer with the purpose described before. An optical circulator is finally used to direct the reflected light towards the optical power meter that will integrate the optical power in a wide region of the spectrum and hence will be able to compute the variation, obtaining refractometric measurements. 


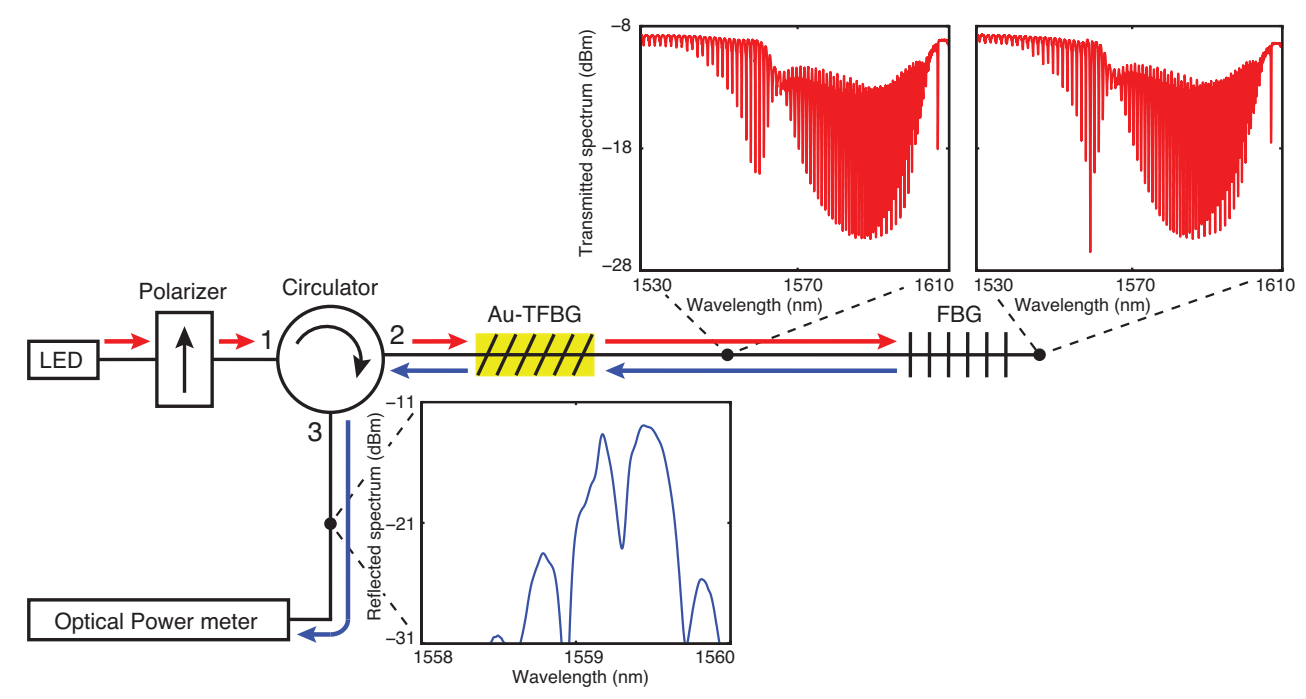

Figure 2. Experimental setup of the reflected power-based technique, with details of the spectrum measured in three different points along the optical path.

\section{RESULTS}

The refractive index of the solution in which the sensor was immersed was changed by modifying its concentration and monitored with a digital refractometer (Reichert AR200). Plasmonic TFBG sensors are usually applied to measure tiny variations in the outer medium, so the solution was slightly modified in such a way that its refractive index changed in an approximate range of $26 \times 10^{-4} \mathrm{RIU}$ (refractive index units). The fabricated sensor was tested in these conditions by both interrogation techniques and a comparative analysis was carried out in order to evaluate their relative performances.

The sensor response with respect to each interrogation technique is illustrated in Figure 3. It exhibits a linear behavior for this refractive index range, as it was expected due to the characteristics of these sensors. A quantitative analysis provides sensitivities of $-2212 \mathrm{~dB} / \mathrm{RIU}$ and $-61 \mathrm{~dB} / \mathrm{RIU}$ and expected resolutions of $4.5 \times 10^{-6}$ RIU and $1.6 \times 10^{-4}$ RIU with the TLS and LED approaches respectively. For the first technique the TLS is extremely narrow, so that the changes of power affect the whole region of the spectrum where the power is confined. As the noise level is sufficiently low, the value integrated by the power meter is assumed to be the optical power value of the laser beam. In addition, the cladding mode resonance not only suffers a power change but also a wavelength shift due to the outer refractive index change. As soon as this shift is produced, the laser begins to point to the edge of the mode, that has a severe slope, and hence the power changes are even higher. However, the second technique relies on the optical spectrum reflected by a FBG, which is known to have a main lobe but also lower power side lobes, also integrated by the power meter. As the sensor most sensitive mode only attenuates a portion of the main lobe, the changes in power are not so significant and comprise just a fraction of the total integrated optical power. That is why the difference between the two techniques in terms of sensitivity is high, although the power change produced in the sensitive mode is the same.

Furthermore, there are some features worth to highlight to compare the two techniques. First, the TLS-based method is not able to differentiate if a power change has been due to a refractive index alteration or to a temperature variation. Since the interrogation is carried out just for the most sensitive mode, if temperature changes, this mode will exhibit a wavelength shift that will ruin the refractometric measurement. However, both the Bragg mode of an FBG and the cladding modes of a plasmonic TFBG sensor have the same temperature sensitivity, that being around $10 \mathrm{pm} /{ }^{\circ} \mathrm{C}$. For this reason, the LED-based technique can be considered to present a temperature-insensitive behavior. Furthermore, to protect the interrogation system from a possible power fluctuation arisen in the light source, the second method could be improved by self-referencing techniques using a second detector ${ }^{9}$. In second place, the use of a LED source and the possibility to substitute the power meter by a photo-diode together with an analog-to-digital converter turns the second technique into a cost-effective solution. And to conclude, this same technique has the advantage of performing measurements based on the reflected spectrum, so a single side of the optical path is required for remote interrogation of the sensor. Hence, the choice of one technique or the other will depend on the requirements of the target application. 

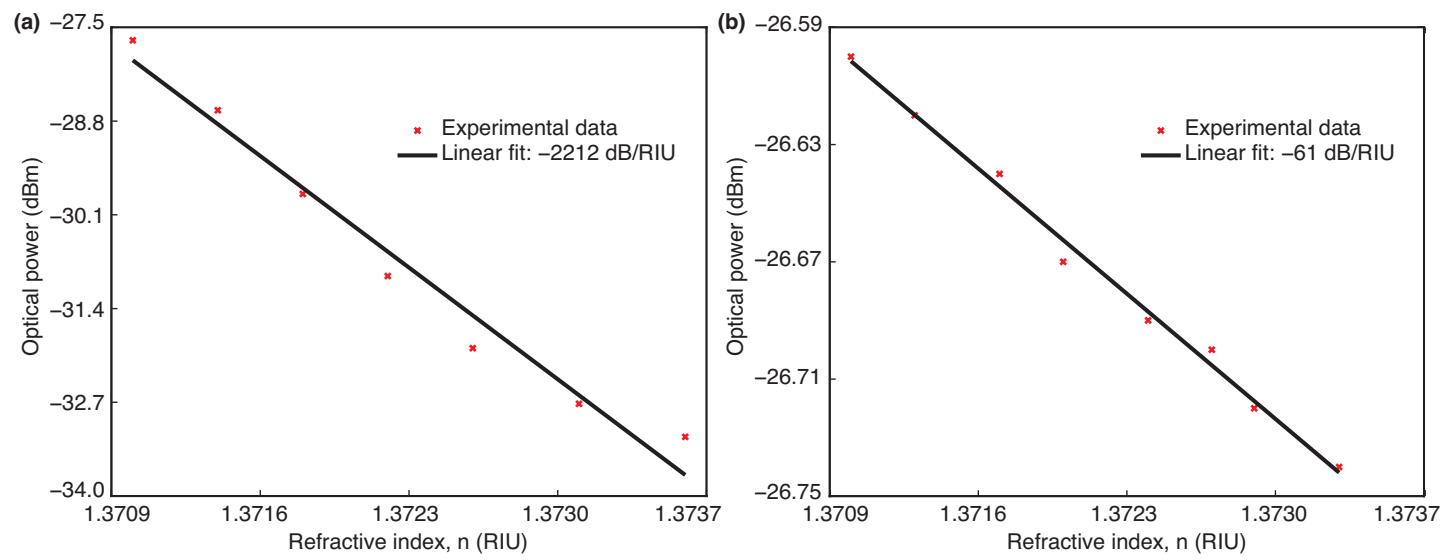

Figure 3. Sensitivity obtained (a) with the tunable laser source configuration and (b) with the LED approach.

\section{CONCLUSION}

Two interrogation techniques for plasmonic TFBG sensors are reported. The first one relies on the transmitted optical power and is characterized by a high sensitivity for refractive index measurements in a defined range. The second method uses the light reflected by a uniform FBG to interrogate the transmitted response of the sensor backwards, comprising a temperature-insensitive and cost-effective solution, also suitable for remote measurements. These two interrogation techniques extend the possibilities to evaluate the performance of this kind of optical sensors.

\section{ACKNOWLEDGEMENTS}

Á. González-Vila is supported by the F.R.S.-FNRS through a FRIA grant. C. Caucheteur is supported by the F.R.S.FNRS. The authors would also like to thank the financial support from the ERC (European Research Council) Starting Independent Grant PROSPER (grant agreement No. 280161 - http://www.umons.ac.be/erc-prosper), from the Spanish Comisión Interministerial de Ciencia y Tecnología within projects TEC2016-76021-C2-1-R and TEC2013-47264-C2-2$\mathrm{R}$ and from SUDOE ECOAL-MGT and FEDER funds from the European Union.

\section{REFERENCES}

[1] Gwon, H. R. and Lee, S. H., "Spectral and Angular Responses of Surface Plasmon Resonance Based on the Kretschmann Prism Configuration," Materials Transactions 51(6), 1150-1155 (2010).

[2] Albert, J., Shao, L. Y. and Caucheteur, C., "Tilted fiber Bragg grating sensors," Laser \& Photonics Reviews 7(1), 83-108 (2013).

[3] Zhou, W., Mandia, D. J., Barry, S. T. and Albert, J., "Absolute near-infrared refractometry with a calibrated tilted fiber Bragg grating," Optics Letters 40(8), 1713-1716 (2015).

[4] Ribaut, C., Voisin, V., Malachovská, V., Dubois, V., Mégret, P., Wattiez, R. and Caucheteur, C., "Small biomolecule immunosensing with plasmonic optical fiber grating sensor," Biosensors and Bioelectronics 77, 315-322 (2016).

[5] Qiu, X., Chen, X., Liu, F., Guan, B. O. and Guo, T., "Plasmonic Fiber-Optic Refractometers Based on a High Q-Factor Amplitude Interrogation," IEEE Sensors Journal 16(15), 5974-5978 (2016).

[6] Caucheteur, C., Voisin, V. and Albert, J., "Near-infrared grating-assisted SPR optical fiber sensors: design rules for ultimate refractometric sensitivity," Optics Express 23(3), 2918-2932 (2015).

[7] Xie, Q., Hong, M. H., Tan, H. L., Chen, G. X., Shi, L. P. and Chong, T. C., "Fabrication of nanostructures with laser interference lithography," Journal of Alloys and Compounds 449(1), 261-264 (2008).

[8] Caucheteur, C., Mégret, P. and Cusano, A., "Tilted Bragg grating multipoint sensor based on wavelength-gated cladding-modes coupling," Applied Optics 48(20), 3915-3920 (2009).

[9] Cusano, A., Cutolo, A. and Albert, J., [Fiber Bragg grating sensors: recent advancements, industrial applications and market exploitation], Bentham Science Publishers, 78-98 (2011). 